\title{
COMpeTitividad Y SUSTENTABILIDAD
} EN EMPRESAS TURÍSTICAS GASTROHOTELERAS: APLiCACión EN CiUdad GuZMáN, JALISCO

\author{
Carlos Hernández Vega \\ Pedro Pablo Villafania Góngora \\ Gonzalo Hernández García \\ Universidad de Guadalajara
}

RECIBIDO: 13 DE MAYO DE 2017 ACEPTAdo: 8 DE Agosto de 2017

\section{RESUMEN}

Ce presentan resultados de una investigación aplica-

$\bigcup$ da sobre el estado actual que guardan las empresas del ramo turístico gastrohotelero en la población de Ciudad Guzmán, Jalisco, en donde se evidencia una creciente demanda de servicios de calidad en hospedaje, alimentación y diversión. El área de estudio es una de las principales ciudades del estado por su factibilidad como polo de desarrollo industrial, comercial y turístico. Se localiza a una hora de Guadalajara, a 47 minutos de la ciudad de Colima, y a 110 minutos de Manzanillo, puerto de entrada y salida a los mercados de la cuenca del Pacífico. Esta localidad tiene en promedio 124 establecimientos de servicios de alimentos y bebidas y hospedaje, ubicados en diversos puntos; sitios donde se ofrecen vinos, destilados y licores (Hernández, 2014). La base principal de la economía en la ciudad está en el sector terciario, el cual representa aproximadamente el $58.2 \%$ de la población activa ( $\mathrm{H}$. Ayuntamiento Zapotlán el Grande, 2017). Esto indica que se ha generado un crecimiento acelerado de restaurantes y algunos de servicio de hospedaje en los últimos cinco años, como respuesta al 
incremento de visitantes, sin embargo, no todos cuentan con las condiciones necesarias para satisfacer la demanda presente ni futura.

Palabras clave: Competitividad, sustentabilidad y turismo.

The results of an applied research on the current status on companies of gastrohotel tourism sector in Ciudad Guzmán, Jalisco, where there is evidence of an increasing demand for quality services in lodging, feeding and entertainment. The study area is one of the main cities of the state due to its feasibility as a pole of industrial, commercial and tourist development. It is located one hour from Guadalajara, 47 minutes from the city of Colima, and 110 minutes from Manzanillo, the port of entry and exit to the markets of the Pacific basin. This locality has an average 124 food, beverage and lodging services establishments, located in different points; sites where wines and liquors are offered (Hernández, 2014). The main base of the economy in the city is in the tertiary sector, which represents approximately $58.2 \%$ of the active population (H. Ayuntamiento
Zapotlán el Grande, 2017). This has generated an accelerated growth of restaurants and some hosting services in the last five years in response to the increase in visitors, however, not all have the necessary conditions to meet current or future demand.

Keywords: Competitiveness, sustainability and tourism.

\section{INTRODUCCIÓN} 1 presente artículo suren el trabajo de investigación "Potencialidad turística del municipio de Zapotlán el Grande, en función de la Sustentabilidad 2013-2015", en donde se evidencia entre otras cosas, una creciente demanda de servicios de calidad en hospedaje, alimentación y diversión por parte de los visitantes a Ciudad Guzmán, Jalisco, misma que es una de las principales urbes del estado por su factibilidad como polo de desarrollo industrial, comercial y turístico.

El crecimiento acelerado de restaurantes y servicios de hospedaje (algunos ligados), en los últimos cinco años, evi- dencia un creciente movimiento de visitantes, los cuales se pueden dividir entre turistas y excursionistas, que en ambos casos, son consumidores; los visitantes turistas en hospedaje y alimentos, y los visitantes excursionistas solo en alimentos.

Con base en lo anterior surge la necesidad de indagar de manera metódica sobre las condiciones de competitividad y sustentabilidad que guarda la ciudad en función de su crecimiento turístico, con el fin de proponer acciones que permitan la toma de decisiones en la creación, ampliación o modificación de más empresas de esta índole y sobre todo cuál es su índice de competitividad entre ellas mismas y las de los destinos turísticos cercanos.

\section{DESCRIPCIÓN} DEL PROBLEMA

n Ciudad Guzmán se
ha presentado un creci-
miento del movimiento de visitantes en los últimos años, tanto de turistas como de excursionistas, quienes cada día demandan más y mejores servicios de calidad en hospedaje, alimentación y diversión; a manera de respuesta, la oferta de servicios que ahora 
mismo están demandando los visitantes también ha crecido, por lo que surge la urgente necesidad de analizar cuáles son las condiciones de competitividad y sustentabilidad en las que se encuentra la ciudad para poder satisfacer las necesidades de los visitantes; de igual manera, de la población local, con el fin de desarrollarse en concordancia con los principios de sustentabilidad que exige la sociedad en términos de responsabilidad social empresarial (RSE).

\section{JUSTIFICACIÓN}

on los resultados de
la presente investi-
gación se pretende generar propuestas de mejora a las empresas dedicadas al servicio de hospedaje, alimentación y diversión en beneficio de los visitantes, así como de la propia población en función de la competitividad y con principios de sustentabilidad, contribuyendo con esto al fortalecimiento del desarrollo turístico en la ciudad.

Además de las ideas de mejoras planteadas, también se podrá contar con un análisis de la situación en la que se encuentran los establecimientos mencionados, cuyos resultados sirvan de base en la creación, ampliación o modificación de empresas.

\section{Pregunta de} INVESTIGACIÓN

$7 \mathrm{n}$ virtud del evidente 4 crecimiento de visitantes en Ciudad Guzmán y sus demandas de atención de calidad en los servicios de hospedaje, alimentación y diversión, ¿los prestadores de servicios turísticos cuentan con las condiciones de competitividad y sustentabilidad suficientes?

\section{Objetivos de INVESTIGACIÓN}

\section{Objetivo}

GENERAL

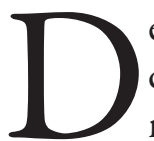
eterminar y evidenciar las condiciones actuales de las empresas gastrohoteleras en Ciudad Guzmán, Jalisco, en función de su índice de competitividad y principios de sustentabilidad con el fin de proponer acciones de mejora para las actividades turísticas.

\section{Objetivos} ESPECÍFICOS

$\sim_{c o s}$ onocer e identificar el total de las empresas del ramo turístico gastrohotelero de Ciudad Guzmán, Jalisco.

Analizar las condiciones que guardan en la actualidad las empresas restauranteras y hoteleras de Ciudad Guzmán, en función de su índice de competitividad y aplicaciones de principios de sustentabilidad.

Proponer acciones concretas de mejora para la actividad turística a partir de las empresas del giro de hospedaje y el de alimentos y bebidas, basadas en la competitividad y la sustentabilidad.

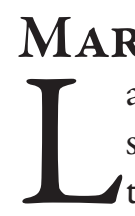

Marco TeÓrico

as teorías básicas que sustentan el presente trabajo se centran en cuatro variables: el turismo, los establecimientos de servicios de hospedaje, alimentación y diversión, la competitividad y la sustentabilidad.

\section{Turismo}

1 turismo en fechas ac-
tuales ha sido conside-
rado como actividad 
tiempo libre, con fuertes repercusiones en lo social y lo ambiental (Hernández, 2017). Este fenómeno socioeconómico cuenta con diversas vertientes en su forma de trabajo:

a) En primera instancia, se debe a los espacios de tiempo libre de las personas, las cuales, a mayor disponibilidad de tiempo tienen también más oportunidad de viajar, siempre y cuando existan las condiciones económicas.

b) En segundo lugar, el movimiento turístico obedece su estructura a la oferta y la demanda. De esta forma la demanda se compone por el total de turistas que efectivamente demandan los servicios turísticos de cualquier sitio y en cualquiera de las modalidades, ya sea de turismo tradicional o turismo alternativo.

Mientras que la oferta turística se puede clasificar en oferta básica y oferta complementaria. En ese sentido, la oferta básica se compone del total de atractivos turísticos, estos de acuerdo con Boullón
(2004), se clasifican en cinco categorías:

- Sitios naturales

- Manifestaciones culturales

- Folklore Realizaciones técnico científicas y contemporáneas

- Acontecimientos programados

Y la oferta complementaria se suma a la básica con todos los servicios necesarios para que el turista pueda realizar su viaje de turismo con satisfacción, estos servicios recaen directamente en el ámbito empresarial y se clasifican entre otras en:

- Empresas de alojamiento

- Empresas de transportación

- Empresas de entretenimiento

- Empresas de alimentos y bebidas conocidos como restaurantes

ESTABLECIMIENTOS DE ALIMENTOS Y BEBIDAS Y HOSPEDAJE

T as empresas de alojamiento y/o alimentos $1 y$ bebidas conocidas como restaurantes y/o bares forman parte muy significativa en el proceso de prestación de servicios al turista en virtud de que algunas de ellas pueden llegar a convertirse en doble propósito para el turista, por un lado, tomarla como es en su concepto básico de oferta complementaria, es decir, cuando el turista acude a un destino con el propósito de visitar un atractivo turístico y de pasada hospedarse o alimentarse; por otro lado, dejar de ser solo oferta complementaria para pasar a ser básica, es decir, cuando el turista acude a un destino con el propósito principal de visitar, en primera instancia, cierto restaurante o bar, y de paso disfrutar de algunos atractivos cercanos. En ese sentido, los establecimientos mencionados forman parte de las condiciones de competitividad con que cuenta un destino turístico.

Competitividad artiendo del principio
general de que en turis-
mo la competitividad es la capacidad de generar sistemáticamente mayores beneficios a la sociedad y rentabilidad a los empresarios de manera permanente, respecto a la 
actividad de referencia en que se compite, surgen múltiples conceptos que explican los alcances del tema.

De acuerdo con Aragón y Rubio (2006), los factores de éxito competitivo de una empresa dependen principalmente de su capacidad para conseguir una posición competitiva favorable, mantener y aumentar su posición en el mercado y con ello obtener constantes resultados superiores sin necesidad de recurrir a una remuneración anormalmente baja de los factores de producción.

Para el Instituto Mexicano de la Competitividad (IMCO, 2005), una empresa llega a ser competitiva cuando logra desarrollar productos y servicios, cuyos costos y calidades son equiparables o mejores a los de sus competidores en el resto del mundo; es por ello que la competitividad, en el ámbito de los servicios gastrohoteleros está sujeta a una mejor rentabilidad que sus competidores, sin dejar de lado la capacidad de satisfacer las necesidades y expectativas de los comensales y huéspedes a los cuales sirve, en función de su misión específica para la cual fue creada.

\section{SUStentabilidad}

$7 \mathrm{n}$ la actualidad, la sus4 tentabilidad o desarroLllo sustentable es uno de los elementos con mayor presencia en los discursos del ámbito político, económico, social, cultural y académico de nuestros días. Es para muchos el tema de moda porque es uno de los paradigmas más orientados a mitigar los efectos que el capitalismo y la globalización están generando.

De acuerdo al documento generado por los acuerdos internacionales, se define al desarrollo sostenible como "Desarrollo que satisface las necesidades de la generación presente, sin comprometer la capacidad de las generaciones futuras de satisfacer sus propias necesidades" (Unesco, 2017).

La palabra desarrollo sustentable o desarrollo sostenible ha creado confusiones conceptuales de raíz que han repercutido en discusiones de diversos organismos y pensadores. En el informe Brundtland se señala que el concepto de "desarrollo sostenible" no se refiere a una estabilidad, fija, de armonía, si no a situaciones de cambio: por un lado, se resalta el carácter dinámico del concepto de desarrollo y por otra, se reconoce la existencia de conflictos y de desequilibrios que son en sí mismos reflejo de situaciones cambiantes y dinámicas.

$\mathrm{Al}$ respecto del concepto, Bifani (1993), consultor de la Organización de las Naciones Unidas, comenta que el cuestionamiento semántico fue superado por un acuerdo sobre cual concepto usar y también el uso indistinto de ambas palabras, sustentabilidad y sostenibilidad, argumentando que estos términos no significan lo mismo, por lo que la confusión deriva de la traducción del idioma inglés. Por ello, Bifani sostiene que la expresión inglesa sustainable development introduce en su versión española un elemento de distinción. En español significa conservar una cosa en su ser o estado, lo cual da idea de un esfuerzo para evitar que una cosa no caiga, en tanto que en la expresión inglesa significa además, avanzar continuamente, mantener la marcha y se refiere a un proceso cuyo ritmo hay que mantener, con lo cual expresa una concepción dinámica. En términos empresariales, 
la sustentabilidad se aterriza en los trabajos de RSE.

RSE es un término que es dinámico y se encuentra en desarrollo, por lo tanto, definirlo resulta complejo. Por lo que se puede caracterizar, según Rendueles y Dreher (2007), para su mejor comprensión integral, gradual y proporcional, es decir, abarca a un conjunto complejo de dimensiones de la empresa, se presenta como un camino de excelencia a seguir y la expectativa de su ejercicio, además su aplicación tiene una relación directa con el tamaño de la empresa, así como, su correspondiente poder o capacidad de influencia en el mercado.

Es conveniente mencionar, de igual forma, la importancia del origen ético de la RSE, que tradicionalmente se pensaba en el mundo empresarial como un ente independiente de la esfera moral y que las reglas económicas eran diferentes a las morales, sin embargo, las partes interesadas de la empresa han roto con esta disociación y exigen que la empresa haga compatible los beneficios económicos con una conducta basada en sóli- dos valores éticos (Rendueles y Dreher, 2007).

Así pues, la RSE se define como el compromiso consciente y congruente de cumplir integralmente con la finalidad de la empresa, tanto en lo interno como en lo externo, considerando las expectativas económicas, sociales y ambientales de todos sus participantes, demostrando respeto por la gente, los valores éticos, la comunidad y el medio ambiente, contribuyendo así a la construcción del bien común (Cajiga, 2004).

Los indicadores concernientes a la RSE consideran un grupo de variables que tienen en cuenta los parámetros sociales, institucionales, económi$\cos$ y ambientales. La evaluación de empresas en términos de la RSE está dividida en siete temas: valores y transparencia, público interno, medio ambiente, proveedores, consumidores, comunidad y gobierno y sociedad (Montes, 2008).

\section{Metodología}

\section{TIPO Y DISEÑO DE INVESTIGACIÓN}

T a investigación tuvo un alcance exploratorio, convirtiéndose luego en descriptiva, con enfoque mixto, de acuerdo con los principios propuestos por Sampieri (2007), donde se expone que la investigación exploratoria es cuando el objetivo es examinar un tema o problema de investigación poco estudiado, del cual se tienen muchas dudas o no se ha abordado antes. Para ello se inició la investigación exploratoria porque no se han encontrado estudios sobre competitividad y sustentabilidad empresarial en la ciudad.

Sampieri (2007) señala además que los estudios exploratorios sirven para preparar el terreno y anteceden a investigaciones con alcance descriptivo, por ello, es posible que una investigación inicie como exploratoria y después sea descriptiva.

\section{Hipótesis}

as empresas dedicadas
al servicio de alimen-
tación diversión en
Ciudad Guzmán, Jalisco re- 
quieren de un mayor índice de competitividad y principios de sustentabilidad para satisfacer la actual demanda de los visitantes y población local.

\section{FASES}

\section{METODOLÓGICAS}

\section{Método}

$\lceil$ r

nvestigación documental y de campo; inventariado

físico de cada uno de los establecimientos y aplicación de encuestas cerradas a los empresarios. La encuesta se aplicó a 86 establecimientos de los 130 registrados en la ciudad, utilizando el criterio de la disponibilidad de los empresarios para responder las preguntas, ubicación céntrica o cercana a los atractivos turísticos y lugares con visitantes mixtos.

\section{INSTRUMENTO}

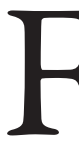

Tormatos específicos $\checkmark$ para el inventario de C las empresas y cuestionario. Se realizó una investigación documental sobre lo ya escrito, lo cual evidenció la necesidad de acudir al campo a levantar los datos de primera mano, por lo que se diseñó un instrumento de 41 preguntas divididas en tres apartados (datos generales, estructura organizacional y planeación estratégica).

\section{ANÁlisis INTERPRETACIÓN DE RESULTADOS \\ Ce utilizó la herramienta - estadística SPSS, con $\bigcup$ apoyo del programa Ex-} cel. A continuación, se describen los resultados generales de cada una de las preguntas.

\section{GIRO DE LA EMPRESA}

n Ciudad Guzmán, Ja-
lisco se cuenta con di-
versidad en cuanto al giro de empresas gastronómicas, que van desde fondas hasta restaurantes bar y hoteleras, extendiéndose en hoteles de 1 hasta 5 estrellas. De todas ellas el $70 \%(n=60)$ son microempresas y el resto están catalogadas como medianas y un mínimo de grandes. Aspecto que coincide con la media nacional especificada por la Encuesta Nacional sobre Productividad y Competitividad de las Micro, Pequeñas y Medianas Empresas (Enaproce), en donde se menciona que del total de empresas hasta el año 2015, $97.6 \%$ son microem- presas y concentran el $75.4 \%$ del personal ocupado total. Le siguen las empresas pequeñas, que son un $2 \%$ y tienen el $13.5 \%$ del personal ocupado. Las medianas representan 0.4 $\%$ de las unidades económicas y tienen poco más del $11 \%$ de los ocupados.

De las empresas encuestadas el $34 \%$ del total, equivalente a 29 , son restaurantes, otro $17 \%(n=15)$ corresponde a "restaurantes bar" donde además de la venta de alimentos, se busca el maridaje, combinando con los platillos el consumo de vino, aunque también se venden bebidas alcohólicas como destilados, distintos estilos de cerveza artesanal, bebidas preparadas, entre otros. Un $5 \%(\mathrm{n}=4)$ corresponde a aquellas empresas que son exclusivamente bares. Además, se cuenta con un 12 $\%(\mathrm{n}=10)$ de cafeterías con venta de postres y helados.

Haciendo alusión a algunos de los protagonistas de la gastronomía mexicana, el 6 $\%(\mathrm{n}=5)$ son taquerías y un $5 \%(\mathrm{n}=4)$ son birrierías, la mayoría de ellas se encuentra en el mercado municipal o a sus alrededores, la birria se ha convertido en un elemen- 
to gastronómico importante para Ciudad Guzmán, sede de la Feria Nacional de la Birria que actualmente cuenta con 3 ediciones. Otro giro que conserva la cultura culinaria de la gastronomía son las fondas, correspondientes a $2 \%(\mathrm{n}=$ 2) de las empresas, en donde se cocina en su mayoría al momento, comida mexicana casera. Por otro lado, un $2 \%(\mathrm{n}=$ 2), corresponde a loncherías.

Existen muchas empresas gastrohoteleras en Ciudad Guzmán y estas se involucran con el turismo, solo el $16 \%$ (n $=14)$ son hoteles, y $1 \%(\mathrm{n}=$ 1) agencias de viajes.

\section{Cargo del} ENTREVISTADO

l momento de realizar
las encuestas, el $33 \%$
$(\mathrm{n}=28)$ de los negocios fueron atendidos por sus propietarios, esto indica que son quienes están de manera constante a cargo de la empresa, el $24 \%(n=21)$ delega las responsabilidades contratando a un administrador o gerente, y el $36 \%(n=31)$ es atendida por encargados, lo que orienta a pensar que son empresas de pocos empleados, pero que constantemente se encuentran supervisadas por el dueño.

\section{ANTIGÜEDAD DEL ENTREVISTADO

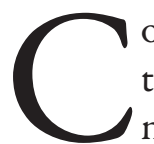 \\ on respecto a los en- trevistados, mencio- nados con anteriori-} dad, la mayoría de ellos tienen más de dos años en la empresa, consistiendo el $52 \%(\mathrm{n}=45)$. El $26 \%(\mathrm{n}=22)$ tiene de uno a dos años. En suma, el $78 \%$ $(\mathrm{n}=67)$ tiene mínimo un año. El otro $22 \%(\mathrm{n}=19)$ tiene menos del año de antigüedad.

\section{GRADO DE ESTUDIO DEL} ENTREVISTADO

oco más de la mitad (54

$\%$, equivalente a 46 )

1 cuenta con estudios inferiores a la licenciatura, y un $43 \%(\mathrm{n}=37)$ tiene la licenciatura, de las cuales $35 \%$ (n $=30$ ) corresponde a los dueños, lo que nos lleva a pensar en que la iniciativa de negocio parte de personas con preparación media al momento de emprender nuevas empresas en estos giros. Otro $35 \%$ ( $n=$ 30) de los de licenciatura pertenece a los administradores que se han contratado, dando a entender que estas empresas esperan contar con una persona capacitada para hacer el trabajo de la administración de la empresa.

\section{EDAD DEL} ENTREVISTADO

$\neg$ res cuartas partes de las personas entrevistadas $(75 \%$, equivalente a 65 personas) son menores a los 45 años de edad, dividido en el rango de personas que tienen de 25 a 34 años, que consta del $39 \%(n=34)$, seguido de aquellas que tienen de 35 a 44 años, con un porcentaje del $23 \%(n=20)$, casi equivalente al $20 \%(\mathrm{n}=17)$ que corresponde a las personas que integran el rango de 45 a 55 años de edad. El rango con menor población es el de las personas de mayor edad: más de 55 años con el $5 \%(n=4)$, seguido por los más jóvenes, que constituyen el $13 \%$ ( $\mathrm{n}=$ 11) restante, este porcentaje está íntimamente relacionado con el nivel de estudios antes mencionado.

\section{GÉNERO}

a mayoría de las per-
sonas a cargo de las
empresas gastrohote- 
fueron entrevistadas, son del sexo masculino, constituyendo un $64 \%(\mathrm{n}=55)$ del total, y dando lugar solo al $36 \%$ (n =31) para las mujeres, aspecto que permea en la región con respecto a las oportunidades laborales igualitarias.

\section{TAMAÑO DE LA EMPRESA}

La mayoría de las empresas gastrohoteleras en Ciudad Guzmán (70\%, equivalente a 60 empresas) son micros y constan de 1 a 10 empleados, el $28 \%(n=24)$ son empresas pequeñas, que cuentan con un promedio de empleados entre el rango de 11 a 50, y tan solo el $2 \%(n=2)$ son medianas empresas, donde su promedio de empleados consta entre los 51 y los 250 .

\section{ANTIGÜEDAD DE LAS EMPRESAS}

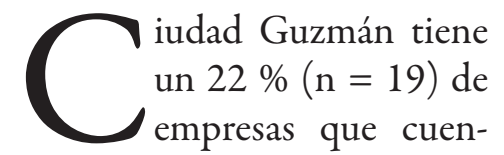
tan con más de 20 años, mismas que aportan identidad, pero también se tiene el surgimiento de nuevas empresas, con un $17 \%(n=15)$ de empresas que tienen menos de dos años en el entorno. El

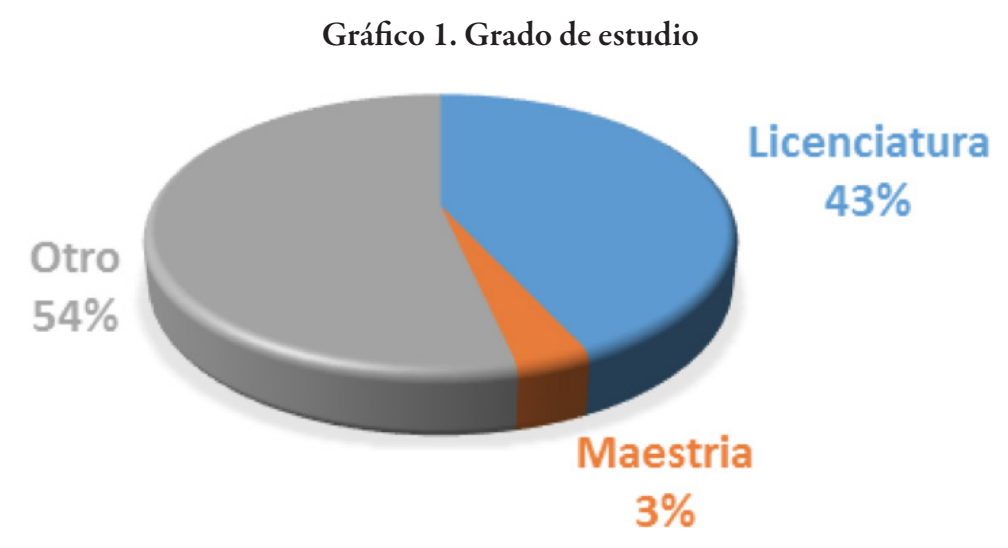

Fuente: Elaboración propia.

Gráfico 2. Tamaño de la empresa

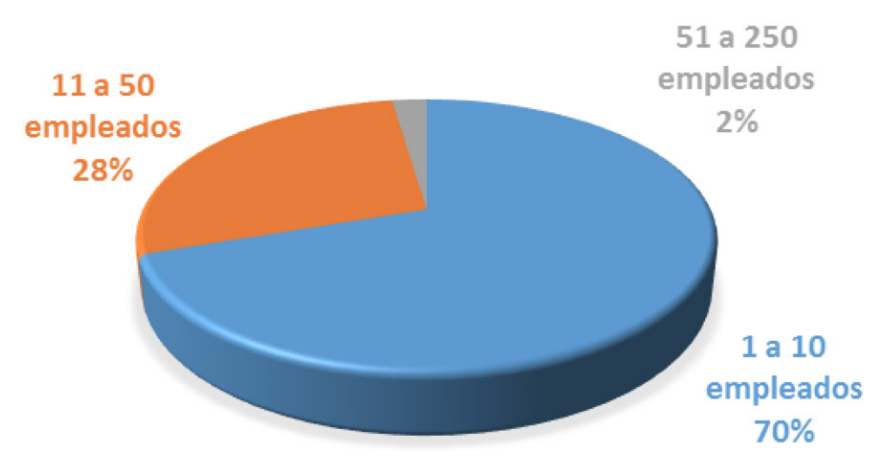

Fuente: Elaboración propia.

rango que acuña la mayor cantidad de empresas es el de 3 a 10 años, el resto de ellas, equivalente al $17 \%(\mathrm{n}=15)$ tiene entre 11 y 20 años brindando servicio en la ciudad.

ESTRUCTURA ORGANIZACIONAL Y DESCRIPCIÓN DE PUESTOS POR ESCRITO a mayoría de las em-
presas gastrohoteleras
en Ciudad Guzmán,
equivalente al $73 \%(\mathrm{n}=63)$,
cuentan con una estructura or-
ganizacional formulada, mien- 
tras que el otro $27 \%(\mathrm{n}=23)$ no lo tiene. Mismos datos que coinciden con los resultados de aquellos que tienen los puestos de sus empresas definidos por escrito, teniendo un $73 \%$ (n $=63$ de un total de 86) de respuesta positiva y el mismo 27 $\%(\mathrm{n}=22)$ anterior de respuesta negativa.

\section{EMPRESAS}

\section{AFILIADAS A ALGUNA} ORGANIZACIÓN RELACIONADA CON EL GIRO DE LA EMPRESA Y ESPECIFICACIONES

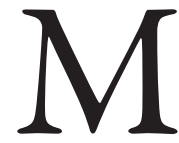
ás de la mitad de las empresas gastrohoteleras de Ciudad Guzmán, equivalente al 65 $\%(n=56)$, no está afiliada a ninguna organización relacionada con el giro de su empresa, mientras el $35 \%(n=30$, de un total de 86) afirma sí estarlo; dentro de este porcentaje, la principal organización manifestada, cumple con un $37 \%(\mathrm{n}=32)$ : la Asociación de Restauranteros y Hoteleros de Jalisco, la siguiente en la lista es la Cámara Nacional de Comercio (CANACO), que contempla al $23 \%(n=20)$, y restaurantes locales y sucursales, al $20 \%(\mathrm{n}=17)$. "CANA$\mathrm{CO}$ y sindicato" y "turismo estatal", son las que tienen el menor porcentaje de empresas afiliadas, con $10 \%(\mathrm{n}=7)$ por cada una.

La principal causa por la que las empresas gastrohoteleras en Ciudad Guzmán no se encuentran afiliadas a alguna organización es porque no lo tienen considerado, con una afluencia del $38 \%(\mathrm{n}=38)$, el $14 \%(n=12)$ expone que eso es decisión exclusiva del dueño y se desconocen las causas que el mismo pueda tener para abstenerse de afiliarse, el $9 \%(\mathrm{n}=$ 8) argumenta ser una empresa independiente. El número de empresas que creen que no las hay, y aquellas que consideran que no ha sido necesario, resultan equivalentes, consistiendo un $7 \%(n=6)$ por cada una. Del mismo modo, pero representando al $5 \%(n=4)$, coinciden aquellas empresas que creen tener sus propias ideas, aquellas que admitieron no tener conocimiento al respecto y las que mencionan que no hay disposición. Por último, las que integran un número menor de empresas son las que aseguran que no resulta beneficioso o es una empresa familiar, consistiendo la más pequeña de las categorías con $4 \%(\mathrm{n}=3)$ cada una.

\section{Alianzas ESTRATÉGICAS CON ALGÚN OTRO TIPO DE NEGOCIO n Ciudad Guzmán, el $42 \%(\mathrm{n}=62)$ de las empresas de giro gas-} trohotelero no cuenta con alianzas estratégicas con algún otro tipo de negocio, solo el 28 $\%(\mathrm{n}=24)$ sí cuenta con ello, de las cuales el $67 \%(n=58)$ afirma que se debe a estrategia comercial y amplitud de servicios, mientras que el $33 \%$ (n $=28$ ) declara ser por beneficio a los empleados. Del $72 \%$ (n =62) que aseguró no contar con este tipo de estrategias, la mitad (50\%, equivalente a 43 de un total de 86), asegura que no hay intereses ni disposición, un $18 \%(\mathrm{n}=15)$ asegura que se debe a que no se ofrecen oportunidades, de manera casi equivalente, el $16 \%(n=14)$ respondió que es decisión de los dueños, así como el $11 \%$ $(\mathrm{n}=9)$ no lo ha pensado y solo el $5 \%(n=4)$ se encuentra en proceso. 
Alianzas ESTRATÉGICAS CON EL MISMO GIRO DEL NEGOCIO

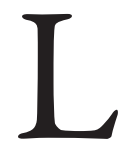

a mayoría de las empresas, no cuentan con alianzas estratégicas en el mismo giro del negocio, representando el $71 \%(\mathrm{n}=61)$, porcentaje del cual, $36 \%(\mathrm{n}=$ 31) se debe a que no lo habían pensado, el $28 \%(\mathrm{n}=24)$ a que no lo consideran viable, el $20 \%(\mathrm{n}=17)$ no sabe o lo desconoce y el $16 \%(n=14)$, al igual que en algunos casos anteriores considera que es decisión exclusiva del dueño.

El $29 \%$ (equivalente 25 de un total de 86) asegura tener alianzas estratégicas con el mismo giro del negocio, teniendo como motivo en un $60 \%(\mathrm{n}=52)$, el usarlo como táctica para brindar un mejor servicio. El otro $40 \%(n=34)$ se distribuye en dos razones: la primera, con el $24 \%(n=21)$ se refiere a que están abriendo más tiendas, y la segunda, representando al $16 \%(\mathrm{n}=$ 14) restante, considera que es complemento para mayor capacidad.

\section{Organiza} EVENTOS ESPECIALES

T $162 \%$ (equivalente a 453 del total de 86) de Llas empresas organizan eventos especiales; dentro de este porcentaje, el $30 \%$ $(\mathrm{n}=26)$ realiza de todo tipo de eventos, el otro $70 \%(\mathrm{n}=$ $60)$ es más especializado, los más comunes son: $28 \%(\mathrm{n}=$ 24) los que se dedican a festividades y $26 \%(\mathrm{n}=22)$ a cumpleaños. Además, el $8 \%$ $(n=7)$ organiza eventos de negocios, un porcentaje más escaso equivalente al $4 \%$ (n =3) organiza desayuno bufé y por último, de forma equitativa está la organización de eventos privados o públicos a empresas con $2 \%(\mathrm{n}=2)$ y noches de karaoke con el $2 \%$ $(\mathrm{n}=2)$ final.

El 38 \% (equivalente a 33) de las empresas afirmó que no realiza este tipo de eventos; de este porcentaje, el $46 \%$ ( $n=$ 40) comenta que es debido a que no lo consideran estrategia, el $30 \%(\mathrm{n}=26)$ a la falta de espacio y tiempo y el $24 \%$ $(\mathrm{n}=21)$ restante reconocen que no ha sido solicitado.
Misión de LAS EMPRESAS

$\neg$ ras realizar las encuestas correspondientes, se descubre que el 62 $\%(\mathrm{n}=53)$ de las empresas gastrohoteleras en Ciudad Guzmán, Jalisco, tienen definida su misión por escrito, porcentaje del cual un $89 \%$ $(\mathrm{n}=77)$ presume que sus empleados la conocen, dejando a un $11 \%(\mathrm{n}=9)$ sin este conocimiento, mientras que, por otro lado el $38 \%(n=33)$ no tiene su misión estipulada en un texto.

\section{Políticas Y PROCEDIMIENTOS \\ 7 asi tres cuartas partes de las empresas gas- trohoteleras en Ciu-} dad Guzmán, es decir, el $74 \%$ $(\mathrm{n}=64)$ presume que sí tiene definidas sus políticas y procedimientos, al menos de forma verbal, porcentaje del cual en un $94 \%$ (81 de las 86 empresas) es difundido por las mismas empresas en sus reuniones de trabajo, dejando lugar solo a $6 \%(\mathrm{n}=5)$ que no hace difusión de ello. El $26 \%(n=22)$ restante no ha definido sus políticas ni procedimientos, ni por escrito ni verbal. 
Gráfico 3. Análisis FODA en las empresas

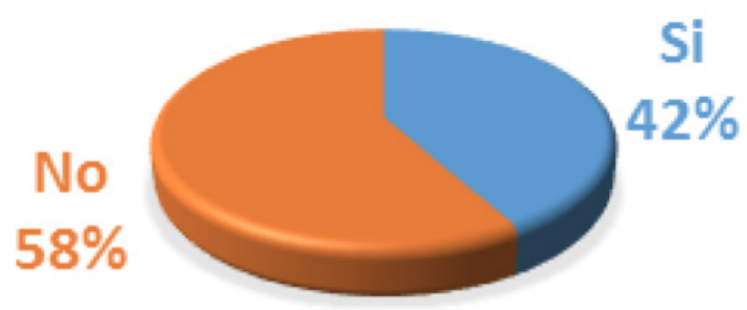

Fuente: Elaboración propia.

\section{Objetivos Y METAS \\ GENERALES}

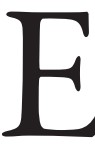

n porcentaje, el $77 \%(\mathrm{n}$ = 66) de las empresas gastrohoteleras en Ciudad Guzmán, dice sí tener objetivos y metas generales establecidos, mientras que el $23 \%$ $(\mathrm{n}=20)$ restante no las tiene especificadas. Del porcentaje que sí los ha definido, es casi equitativo el número de empresas que tiene objetivos para cada una de las áreas funcionales y aquellas que no, es decir $49 \%(\mathrm{n}=42)$ y $51 \%(\mathrm{n}=44)$, respectivamente.

El $70 \%(n=60)$ de las empresas gastrohoteleras en Ciudad Guzmán, asegura tomar en cuenta a su personal para la toma de decisiones de la em- presa, mientras el $30 \%(\mathrm{n}=$ 26) admite que no lo hace.

\section{ANÁlisis FODA EN LA EMPRESA}

D s mayoría el porcenta4 je de empresas que no realiza análisis FODA en los giros gastrohoteleros en Ciudad Guzmán, incluso desconocen el término, correspondiente al $58 \%(\mathrm{n}=50)$, dando así lugar a un $42 \%$ ( $\mathrm{n}=$ 36) a aquellas que sí lo llevan a cabo, de las cuales la mitad, que corresponde al $25 \%$ ( $\mathrm{n}=$ 22) por cada una lo realizan de forma semanal y mensual, el $17 \%(\mathrm{n}=15)$ lo realiza cada 6 meses, mientras que el $14 \%$ (n $=12$ ) lo elabora diariamente, caso equitativo a aquellas que lo realizan cada 3 meses. Por último, el $6 \%(n=5)$ corres- ponde a las empresas que deciden analizar de forma anual.

\section{Estrategias}

PARA ALCANZAR LOS OBJETIVOS PROPUESTOS POR

\section{LA EMPRESA}

$\mathrm{L}$

a mitad (50 \% equivalente a 43 del total de las empresas gastrohoteleras en Ciudad Guzmán plantean estrategias de calidad y servicio al cliente, el $27 \%$ implementa en ellas promociones y capacitaciones, mientras que el $13 \%(n=11)$ se concentra en diagnóstico de evaluación, por otro lado, el $8 \%(\mathrm{n}=7)$ de las empresas ni siquiera tienen estrategias definidas, además un $2 \%$ (n $=2$ ) solo siguen el protocolo previamente establecido.

\section{Proveedores de} MATERIALES

T a mayoría de las empresas, equivalente al $195 \%$ (82 del total de 86), cuentan con más de un proveedor de los diferentes materiales que utilizan para la elaboración de su producto, mientras que solo el $5 \%$ ( $\mathrm{n}=$ 4) se concentra en uno solo, en su totalidad debido a la com- 
paración de precios. Además, solo el $31 \%(n=27)$ de las empresas asegura que sus proveedores son locales, mientras el otro $69 \%(n=59)$, que corresponde a la mayoría, cuenta con proveedores externos debido a diversos factores: el $35 \%(\mathrm{n}=30)$ porque busca mejor calidad, mientras que el $29 \%(n=25)$ es debido a la inexistencia del producto; así como el $23 \%(\mathrm{n}=22)$ es a causa del precio, el $7 \%$ (n $=6$ ) por la facilidad de pago, aunque también se da por la comodidad de entrega, como es el caso del $4 \%(n=3)$; el último $2 \%(\mathrm{n}=2)$ está indefinido.

\section{FinANCiAmiento} EXTERNO

E $177 \%(\mathrm{n}=66)$ de las empresas gastrohoteleras en Ciudad Guzmán, no cuenta con financiamiento externo y aunque es mayoría, el otro $23 \%(n=20)$ sí cuenta con él, de ellos el $40 \%(\mathrm{n}=$ 34) es para bancos y el mismo porcentaje para cajas populares, con un $20 \%(n=17)$ restante para apoyos de otro tipo. De la mayoría que no cuenta con el financiamiento externo, el $65 \%(\mathrm{n}=56)$ no lo consi- dera necesario, mientras el 18 $\%(\mathrm{n}=15)$ mencionó que no hay facilidades ni oportunidades para obtenerlos. El resto, equivalente al $17 \%(\mathrm{n}=15)$, casi equivalente al anterior, comenta que el dueño no lo ha dispuesto y es quien debe hacerse cargo.

\section{Aplicación Del FINANCIAMIENTO

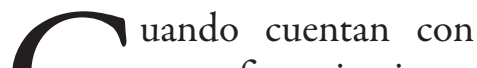 un financiamiento externo, la aplicación} es primordial, y cada empresa lo emplea de modo distinto. En las empresas gastronómicas y hoteleras en Ciudad Guzmán, $56 \%(\mathrm{n}=48)$ lo usa para la compra de material mientras que otro $24 \%(n=21)$ lo requiere para modernización, $16 \%$ (equivalente a 14 del total de 86) para ampliación, así como el $4 \%(\mathrm{n}=3)$ restante lo usa para otros fines no especificados.

\section{Sustentabilidad}

\section{Prácticas de} RESPONSABILIDAD SOCIAL EMPRESARIAL

e las 86 empresas que
se tomaron en cuenta
para la investigación, 61 de ellas, equivalente al 71 $\%$ no realizan prácticas de responsabilidad social empresarial, dando lugar solo a 25 empresas que sí lo hacen, equivalente al $29 \%$. Dentro de estas, el $44 \%(n=11)$, hace uso de productos biodegradables, el $32 \%(\mathrm{n}=8)$ realiza donativos, un $16 \%(\mathrm{n}=4)$ procura el ahorro de energía eléctrica, y por último, solo el $8 \%(\mathrm{n}=2)$ la separación de residuos.

\section{Principios De SUSTENTABILIDAD}

a mayoría de las empre-
sas $(62 \%$, equivalente
a 53 del total de 86$)$, sí trabajan con principios de sustentabilidad, mientras que el $38 \%$ restante $(\mathrm{n}=33)$ no lo hace, porcentaje del cual el $100 \%$ (33 empresas) aseguran que es debido al desconocimiento. El principio de sustentabilidad más común es la separación de residuos, siendo 
25 de las 53 empresas que sí los llevan a cabo, equivalente al $48 \%, 21 \%(\mathrm{n}=11)$ al uso de productos biodegradables, un $17 \%(n=9)$ se enfoca en el ahorro de energía, mientras que el $14 \%(n=7)$ restante no proporcionó información.

\section{Conclusiones}

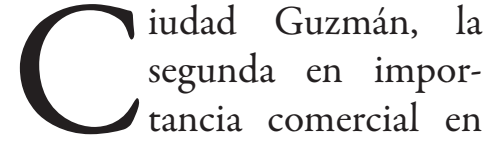

el estado de Jalisco, cuenta con más de 130 establecimientos ligados al servicio turístico gastrohotelero y algunas agencias de turoperadores.

El más alto movimiento comercial de la ciudad se ve en mayor porcentaje de lunes a viernes para el turismo de negocios (personas que viajan por motivos de trabajo), un bajo consumo los fines de semana por turistas del segmento de descanso, además de los habitantes locales y regionales siguiendo la tradición familiar de acudir los domingos a comer a algún establecimiento de alimentos y bebidas.

El tráfico comercial entre semana se presenta por la población flotante de estudiantes y trabajadores de las empresas agrícolas cercanas, mismos que en fines de semana se ausentan y la ciudad vislumbra un vacío.

Este alto movimiento flotante ha creado expectativas a los empresarios para emprender nuevos negocios relacionados al giro en estudio, del cual después de su análisis en campo se concluye que, en términos de competitividad, es necesario mejorar numerosos aspectos que van desde la estructura organizacional improvisada hasta la aplicación de principios de planeación estratégica.

Los empresarios son en su mayoría jóvenes con nivel de estudios medio pero que emplean a personas con estudios universitarios, solo un $22 \%$ se ha mantenido por casi 20 años debido a que realizan innovaciones y son los negocios que corresponden a familias de abolengo, el resto son jóvenes y otras más que no fueron encuestadas, iniciaron y no lograron mantenerse por más de un año.

Las empresas que se han mantenido y que se encuentran en situación creciente, son aquellas que utilizan, sin saberlo, académicamente al cien por ciento, los principios de la administración con bases de competitividad, y además están dispuestos a mejorar aprovechando los apoyos gubernamentales que se les ofrecen, como dato adicional en el periodo de octubre 2016 a mayo 2017 , en la ciudad 80 empresas están recibiendo apoyos de consultoría por medio del Instituto Nacional del Emprendedor (Inadem).

En el tema de sustentabilidad, la gran mayoría desconoce el tema como tal, sin embargo, lo confunde con el hecho de cuidar el medio ambiente y colaborar en navidad con regalos para los niños o algunos con la separación de basura.

Con los resultados obtenidos se ha podido dar a conocer a los empresarios la necesidad de mejorar en términos de competitividad y sustentabilidad para satisfacer la demanda creciente de visitantes y ya se cuenta con apoyo en algunas de las empresas. 


\section{REFERENCIAS}

Aragón, A. y Baños A. (2006, mayo-agosto). Factores explicativos del éxito competitivo: El caso de las PYMES del estado de Veracruz. Revista Contaduría y Administración, 216. México.

Bifani, P. (1993). Desarrollo sostenible, población y pobreza: algunas reflexiones conceptuales. Arturo Curiel Ballesteros (Presidente). Educación ambiental y universidad. Congreso Iberoamericano de Educación Ambiental. Universidad de Guadalajara, México.

Boullón, R. C. (2004). Planificación del espacio turístico. México: Trillas.

Hernández Sampieri, R., Fernández Collado, C. \& Baptista Lucio, P. (2007). Metodología de la investigación. México: McGrawHill.

Hernández Vega, C. (2014). Baco en Zapotlán, consumidores y vendedores de vino en Ciudad Guzmán, Jalisco. México: STAUDG. Virgen Aguilar, C. R. (2014). Turismo y desarrollo sustentable. Un acercamiento al estudio del turismo. Mé- xico: Universidad de Guadalajara.

Red Mundial

Cajiga Juan, F. (2004). El concepto de Responsabilidad Social Empresarial. Centro Mexicano para la Filantropía. Recuperado de: http://www.cemefi.org/ esr/images/stories/pdf/ esr/concepto_esr.pdf

Encuesta Nacional sobre Productividad y Competitividad de las Micro, Pequeñas y Medianas Empresas (2015). Boletín de prensa, 16(285), 1-1 Recuperado de: http://www.inegi.org. $\mathrm{mx} /$ saladeprensa/boletines/2016/especiales/especiales2016_07_02.pdf

H. Ayuntamiento de Zapotlán el Grande (2017). Economía: Aspectos generales. Recuperado de: http:// ciudadguzman.gob.mx/ Pagina. aspx $? \mathrm{id}=628163 \mathrm{bf}-$ 0414-465b-90bd-b2c8b3d558ef

Instituto Mexicano para la Competitividad. (2005). Los retos de la competitividad en México. Una agenda de reformas inmediatas. Recuperado de: http:// cidac.org/esp/uploads/1/
Los_retos_de_la_competitividad_en_M_xico._ Una_agenda_de_reformas_inmediata_PDF.pdf

Mercado S. y García $H$. (2007). La responsabilidad social en empresas del valle de Toluca, México. Un estudio exploratorio. Estudios gerenciales, 23(102), Universidad ICESI, Cali, Colombia. Recuperado de: http://redalyc.uaemex. $\mathrm{mx} / \mathrm{src} /$ inicio/ArtPdfRed. jsp $? \mathrm{iCve}=21210205$

Montes Vásquez, J. (2008). Ecoeficiencia: una propuesta de responsabilidad ambiental empresarial para el sector financiero colombiano (tesis de maestría en medio ambiente y desarrollo. Universidad Nacional de Colombia, sede Medellín.) Recuperado de: http://www.ecobanking. $\mathrm{com} / \mathrm{PT} /$ publicaciones/ instituciones_academicas/ Tesis-JMontes.pdf

Rendueles, M., \& Dreher, M. (2007). La dualidad sujetoobjeto y la responsabilidad social empresarial, 9(1), 115-129. Recuperado de: http://redalyc.uaemex. $\mathrm{mx} / \mathrm{src} /$ inicio/ArtPdfRed. jsp ?iCve $=99314566008$ 


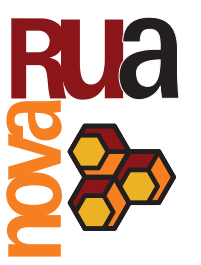

Organización de las Naciones Unidas para la Educación, la Ciencia y la Cultura, Unesco (2017). Desarrollo sostenible. Recuperado de: http://www. unesco.org/new/es/education/themes/leadingthe-international-agenda/ education-for-sustainabledevelopment/sustainabledevelopment/ 